\title{
Shock absorbing characteristics and vibration transmissibility of honeycomb paperboard
}

\author{
Yanfeng Guo ${ }^{\mathrm{a}, \mathrm{b}}$ and Jinghui Zhang ${ }^{\mathrm{a}}$ \\ a School of Architectural Engineering and Mechanics, Xi'an Jiaotong University, Xi'an 710049, Shaanxi Prov., P.R. \\ China \\ ${ }^{\mathrm{b}}$ Department of Packaging Engineering, Xi'an University of Technology, Xi'an, 710048, Shaanxi Prov., P.R. China
}

Received 19 October 2003

Revised 27 November 2003

\begin{abstract}
Honeycomb paperboard is a kind of environmental-friendly package cushioning material with honeycomb sandwich structure, and may be employed to protect products from shock or vibration damage during distribution. This paper deals with the characterization of properties of honeycomb paperboard relevant to its application for protective packaging in transportation, such as dynamic cushion curves and vibration transmissibility. The main feature of the paper is the evaluation on the shock absorbing characteristics and vibration transmissibility of honeycomb paperboards with different thickness by a series of experimental studies on the drop shock machine and vibration table. By using the fitting polynomial of the curve, the experiential formulas and characteristic coefficients of dynamic cushion curves of honeycomb paperboards with different thickness have been obtained. From the vibration tests with slow sine sweep, the peak frequencies and vibration transmissibility are measured and used to estimate the damping ratios. All the works provide basic data and curves relevant to its application for protective packaging in transportation.
\end{abstract}

Keywords: Honeycomb paperboard, shock absorbing characteristics, vibration transmissibility

\section{Introduction}

Honeycomb sandwich structures usually have two layers of thin skins and one thick lightweight core, which hold high strength-to-weight ratio and stiffness-to-weight ratio [6]. Since the early 1940s, aluminum honeycomb sandwich structures have come into wide use in aeronautical industry. A variety of materials besides aluminum alloy are now available as honeycomb structures, such as foams, paper and 'Nomex' honeycombs [12].

In the field of transport packaging, corrugated paperboard and honeycomb paperboard are two kinds of environmental-friendly package cushioning materials with sandwich structures. Corrugated paperboard is a commonly used material to replace plastic foams in transport packaging, mainly due to environmental concerns, and its compressive resistance, shock absorbing characteristics and vibration transmissibility have been studied by Sek and Kirkpatrick et al. $[5,9,10]$.

Honeycomb paperboard belongs to a kind of paperboard with honeycomb sandwich structure. It is made of Kraft linerboard paper, reusable core paper and water-based glue. Because these materials are recyclable, reusable and biodegradable, honeycomb paperboard has economic and environmental advantages over plastic foams. Honeycomb sandwich structure also has lightweight, high strength-to-weight ratio and stiffness-to-weight ratio. Therefore there is an increasing interest in using honeycomb paperboard as structural elements for protective packaging. Examples include precise equipments and instruments, household appliances and fragile goods etc. In the reference [4], compressive resistance of honeycomb paperboard with different thickness has been studied. But the lack of dynamic cushion curves and vibration transmissibility of honeycomb paperboard hampers its application in transport packaging and replacement for plastic foams. So, the aims of this study are as follows: first, to investigate shock absorbing characteristics for a single impact (drop) and present experiential formulas and characteristic coefficients of the dynamic cushion curves of honeycomb paperboards with different thickness. Secondly, to analyze vibration transmissibility, peak frequencies and damping ratios of honeycomb paperboards with different thickness. 


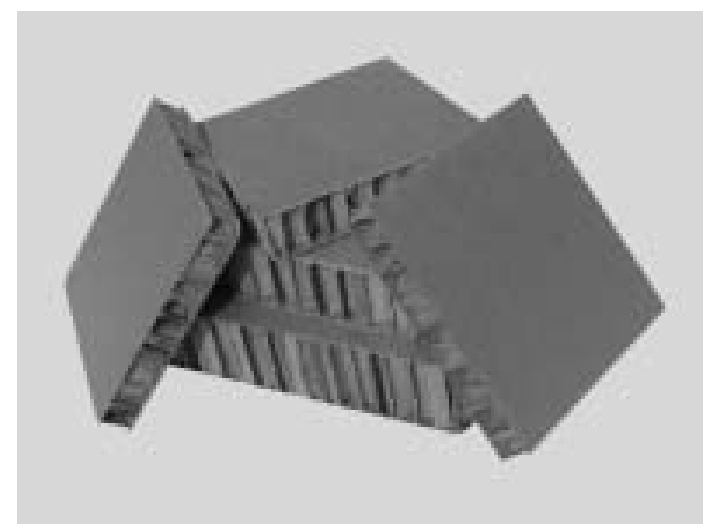

Fig. 1. Photograph of the honeycomb paperboards.

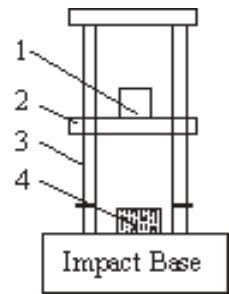

(a)

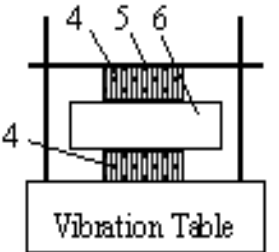

(b)

1.Weight 2. Dropping head 3. Guide column 4. Test specimen

5. Clamp device 6. Mass block

Fig. 2. The mount and fixture of test specimen.

\section{Test specimens and test method}

Experimental materials are honeycomb paperboards with different thickness (see to Fig. 1), and their thickness are 20,30, 40 and $50 \mathrm{~mm}$, respectively. The skins have the same material, thickness and substances, and the core has regular hexagonal cells. The thickness of skins is much smaller than that of the honeycomb core. The skins of the honeycomb paperboards are Kraft linerboard paper with substances of $300 \mathrm{~g} / \mathrm{m}^{2}$, and the core is reusable paper which weight is of $110 \mathrm{~g} / \mathrm{m}^{2}$. Before tests, all the test specimens are preprocessed for twenty-four hours at temperature $21^{\circ} \mathrm{C}$ and relatively humidity $64 \%$.

The shock absorbing characteristics of package cushioning materials is usually presented as a family of dynamic cushion curves. A family of dynamic cushion curves shows peak acceleration $G_{m}$ during impacts for a range of static loads exerted on test specimen, and is constructed for several drop heights. The peak acceleration $G_{m}$ is a non-dimension ratio of peak acceleration of the weight to the gravity acceleration $\mathrm{g}$. The most comprehensive method for determining shock absorbing characteristics of package cushioning materials is described in the ASTM D 1596 Standard [1]. The mount and fixture of test specimen of honeycomb paperboard are shown in Fig. 2 (a). The drop shock machine has a dropping head (to represent a packaged item) and impact base for dynamic loading of a test specimen to simulate impact in rough handling. A transducer is mounted on the drop head, and the signal output from the transducer is fed into a suitable recording system. The reading system should be calibrated to read peak acceleration. The weight is mounted on the dropping head. The mass of the weight and drop height of the dropping head are adjustable.

The vibration transmissibility of package cushioning materials is usually described as the relationship between vibration transmissibility $T_{r}$ and peak frequency $f_{r}$ at different static stress. Vibration transmissibility $T_{r}$ is a non-dimension ratio of response acceleration amplitude of the package system in steady-state forced vibration to 
excitation acceleration amplitude. The most comprehensive method for determining vibration transmissibility of package cushioning materials is described in the ASTM D 4168 Standard [2]. The test apparatus consists of a vibration tester, and the mass block and fixture representing a packaged item are mounted on the vibration tester. The mount and fixture of test specimens are shown in Fig. 2 (b). By changing the weight of the mass block, the static stress exerted on test specimen may be adjusted. The signal outputs from transducers located in the mass block and on the platform of the vibration table are fed into a suitable recording system.

\section{Shock absorbing characteristics of honeycomb paperboard}

In this part, the shock absorbing characteristics of honeycomb paperboards with different thickness is investigated by making tests on the drop shock machine, and the experiential formulas and characteristic coefficients of the dynamic cushion curves are obtained by using the fitting polynomial of curve.

\subsection{Description of shock absorbing characteristics}

In Fig. 2 (a), the weight and dropping head is dropped on the test specimen of honeycomb paperboard from a drop height, the kinetic energy of them is completely stored by the compression of test specimen (neglecting other energy losses such as heat energy). When the maximum deformation $x_{m}$ (or strain $\varepsilon_{m}$ ) of the test specimen is reached, the maximum stress $\sigma_{m}$ and peak acceleration $G_{m}$ would be occurred. According to the law of conservation of energy, the kinetic energy of the weight and the dropping head is equal to the deformation energy of test specimen [8]

$$
A T \int_{0}^{\varepsilon_{m}} \sigma d \varepsilon=W\left(h+x_{m}\right)
$$

where $W$ stands for the weight of the dropping head and weight, and $h$ is the drop height, $\sigma$ is the stress of test specimen, $T$ is the thickness of test specimen, and $A$ is the contact area between the test specimen and dropping head. In a general case, $h \gg x_{m}$, so Eq. (1) may be simplified as

$$
A T \int_{0}^{\varepsilon_{m}} \sigma d \varepsilon=W h
$$

From reference [8], the following expression is referred [8]

$$
\sigma_{m}=G_{m} \sigma_{s}
$$

where $\sigma_{s}$ is the static stress of the test specimen

$$
\sigma_{s}=\frac{W}{A}
$$

Substituting Eqs (3) and (4) into Eq. (2), the relationship between the thickness of test specimen, drop height and peak acceleration may be described as

$$
T=C \frac{h}{G_{m}}
$$

where

$$
C=\frac{\sigma_{m}}{\int_{0}^{\varepsilon_{m}} \sigma d \varepsilon}
$$

It is clear that cushioning coefficient $C$ may be derived from the peak acceleration $G_{m}$ and static stress $\sigma_{s}$. Therefore a dynamic cushion curve (or $G_{m}-\sigma_{s}$ curve) indicates directly the relationship between $G_{m}$ and $\sigma_{s}$ during impacts for a range of static loads and a drop height. A family of dynamic cushion curves may describe the shock absorbing characteristics of package cushioning material, and is constructed for several drop heights. The shape of $G_{m}-\sigma_{s}$ curve also indicates the efficiency of package cushioning material to isolate impact. The lower the curve swings, the better protection the material provides. The curve has proved to be the most practical basis for describing the shock absorbing characteristics of package cushioning material, and may be derived from dynamic compressive test data according to the test procedure described in ASTM D 1596 [1]. 


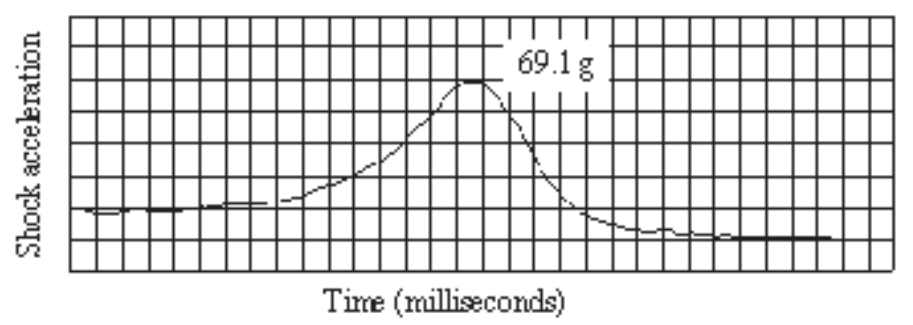

Fig. 3. Shock acceleration-time curve of honeycomb paperboard $(T=40 \mathrm{~mm}, D H=60 \mathrm{~cm})$.

Table 1

Characteristic coefficients of dynamic cushion curves of the honeycomb paperboards

\begin{tabular}{ccl}
\hline $\mathrm{T} / \mathrm{mm}$ & $\mathrm{DH} / \mathrm{cm}$ & \multicolumn{1}{c}{$a_{0}, a_{1}, a_{2}, a_{3}$} \\
\hline 20 & 40 & $259.67,-1326,2477.2,-1354$ \\
& 60 & $386.76,-2450.4,5575.4,-3529.7$ \\
30 & 40 & $220.87,-842.79,997.82,-328.44$ \\
& 60 & $264.49,-995.3,1221.2,-407.37$ \\
& 90 & $361.21,-2215.1,4946.3,-2981.3$ \\
40 & 40 & $199.69,-626.51,633.03,-184.79$ \\
& 60 & $300.54,-1395.7,2119,-954.35$ \\
& 90 & $343.58,-1786.9,3127.5,-1530.7$ \\
50 & 40 & $194.65,-577.88,556.94,-157.87$ \\
& 60 & $315.3,-1578.5,2523.7,-1200.4$ \\
& 90 & $300.82,-1568.1,2648.6,-1232.5$ \\
\hline
\end{tabular}

\subsection{Experiential formulas and characteristic coefficients of dynamic cushion curves}

According to the test method described in the ASTM D 1596 standard [1], the dynamic compressive tests of honeycomb paperboards with different thickness are made on the drop shock machine, and the drop height is selected 40,60 and $90 \mathrm{~cm}$ respectively. Because majority of test specimens of the honeycomb paperboards are locally crushed and the honeycomb cores are locally out of shape after two consecutive impacts, each of test specimens is made only for a single impact (drop) but not for consecutive impacts on the drop shock machine in the procedure of dynamic compressive tests. In addition, the shock absorbing characteristics of the honeycomb paperboards has close relationship with three parameters such as drop height, static stress and the thickness of honeycomb paperboard. So, in the procedure of dynamic compressive tests of the honeycomb paperboards, the three parameters are changed respectively. For example, supposing the drop height and thickness of honeycomb paperboard are constant, the static stress or the mass of the weight is changed, a series of test data and one piece of dynamic cushion curve may be obtained. Therefore by making dynamic compressive tests, the shock absorbing characteristics of honeycomb paperboard for a single impact (drop) may be estimated, and eleven pieces of dynamic cushion curves (which are shown in the following Section 3.3) of honeycomb paperboards with different thickness for the drop heights of 40,60 and $90 \mathrm{~cm}$ are obtained.

The waves of shock acceleration are similar to half-sine pulses in the dynamic compressive test of honeycomb paperboards with different thickness. The wave given in Fig. 3 is that of honeycomb paperboard with thickness $40 \mathrm{~mm}$ for drop height $60 \mathrm{~cm}$ and static stress $1.873 \mathrm{kPa}$.

The shapes of the dynamic cushion curves of honeycomb paperboards with different thickness are always concave and upward, and each piece of the curves has only one minimum value point. For example, the curve given in Fig. 4 is the dynamic cushion curve of honeycomb paperboard with thickness $40 \mathrm{~mm}$ for drop height $60 \mathrm{~cm}$. In Fig. 4, 'T' and 'DH' represent the thickness of honeycomb paperboard and drop height, and the circle dots stand for test data of average peak acceleration. The dynamic cushion curve is obtained by using the fitting polynomial of the curve.

A dynamic cushion curve has close relationship with the thickness of material, the drop height and static stress. The equation to fit dynamic cushion curve and the fitting polynomial of dynamic cushion curve have been studied by 


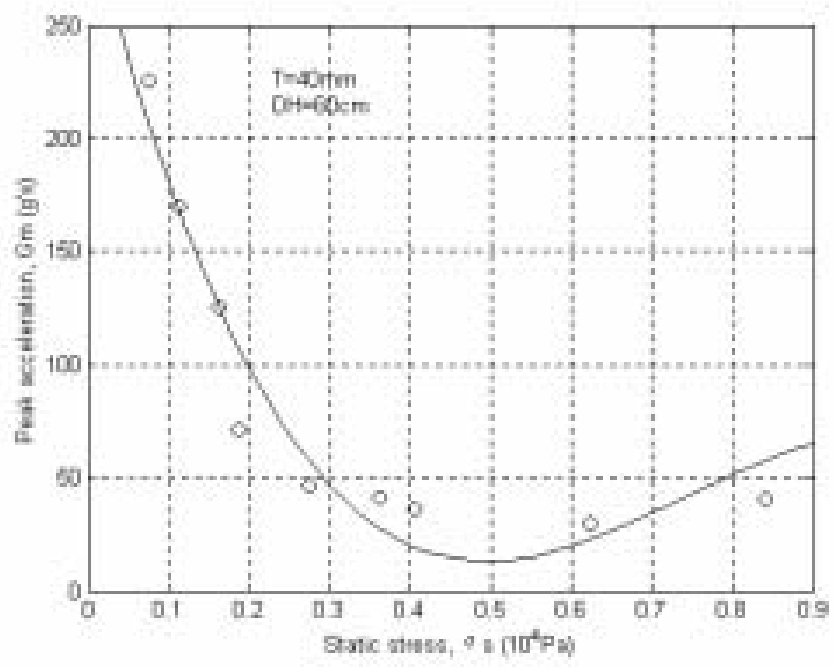

Fig. 4. Dynamic cushion curve of honeycomb paperboard.

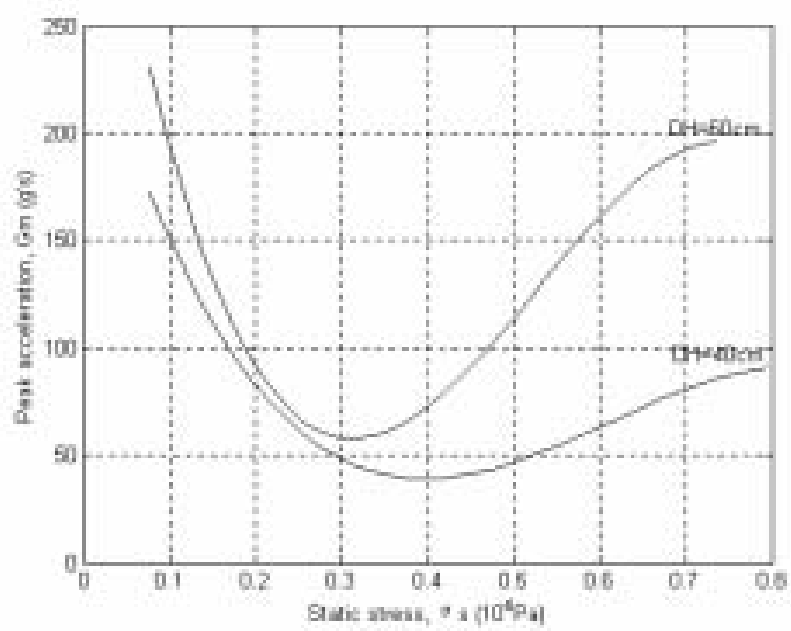

Fig. 5. Dynamic cushion curves of honeycomb paperboard $(T=20 \mathrm{~mm})$.

Burgess [3]. In order to obtain the dynamic cushion curves of honeycomb paperboards with different thickness for different drop heights, the fitting polynomial of the curve is used. At first, according to the shapes of the dynamic cushion curves of honeycomb paperboards with different thickness, supposing that the experiential formulas of the dynamic cushion curves are third order polynomials function written as [3]

$$
G_{m}=a_{0}+a_{1} \sigma_{s}+a_{2} \sigma_{s}^{2}+a_{3} \sigma_{s}^{3}
$$

where $a_{0}, a_{1}, a_{2}$, and $a_{3}$ are four characteristic coefficients to be solved by Method of the Least Mean Square. Then, on the basis of test data obtained from the dynamic compressive tests of honeycomb paperboards with different thickness, using Eq. (7) and Method of the Least Mean Square, characteristic coefficients of the dynamic cushion curves are respectively solved and shown in Table 1. ' $\mathrm{T}$ ' and ' $\mathrm{DH}$ ' given in Table 1 represent the thickness of honeycomb paperboard and drop height. 


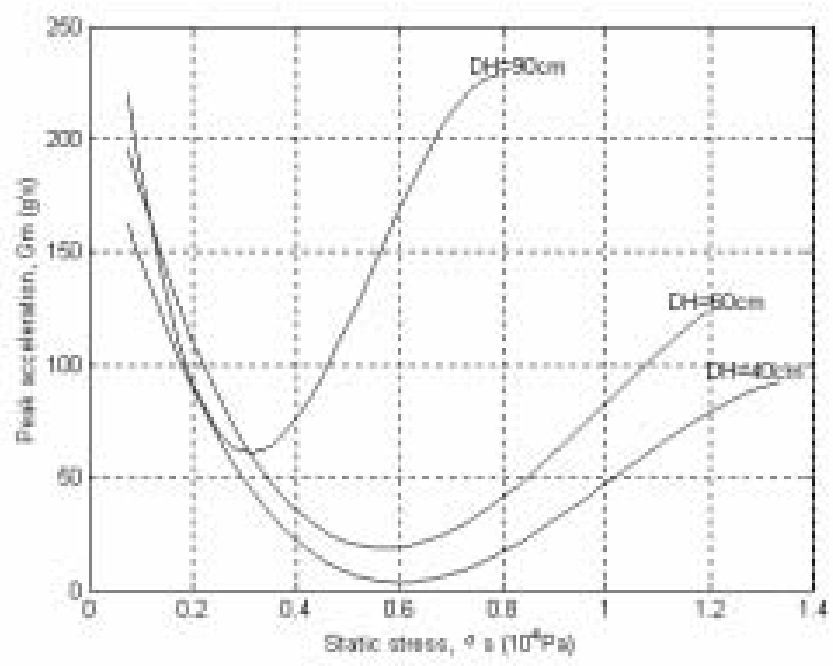

Fig. 6. Dynamic cushion curves of honeycomb paperboard $(T=30 \mathrm{~mm})$.

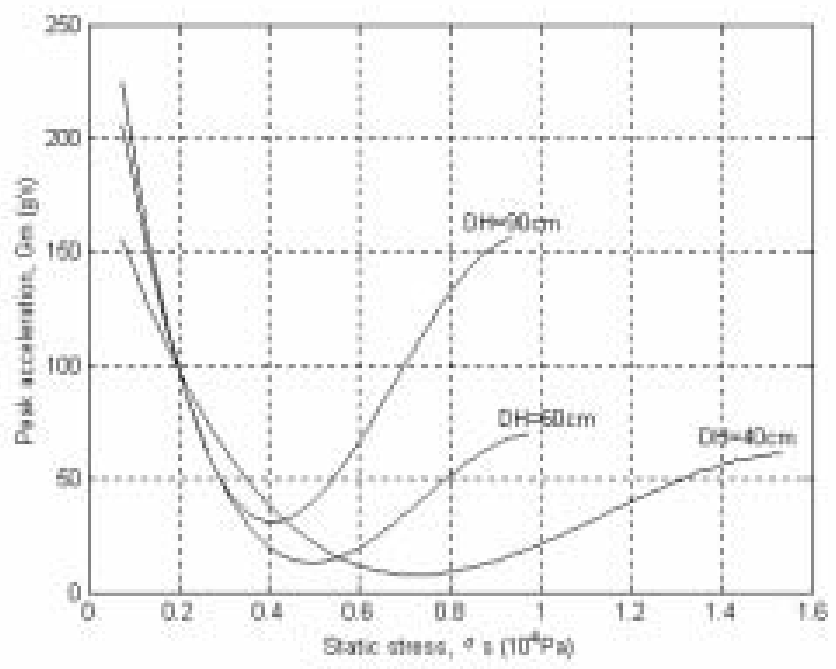

Fig. 7. Dynamic cushion curves of honeycomb paperboard $(T=40 \mathrm{~mm})$.

\subsection{Experimental results}

By making the dynamic compressive tests of honeycomb paperboards with different thickness for the drop heights 40, 60 and $90 \mathrm{~cm}$, eleven pieces of dynamic cushion curves are obtained, which are shown in Fig. 5 to Fig. 11. In each of Fig. 5 to Fig. 8, the thickness of honeycomb paperboard is respectively constant for each figure, yet the drop height is variable. In each of Fig. 9 to Fig. 11, the drop height is respectively constant for each figure, yet the thickness of honeycomb paperboard is variable. Comparing the dynamic cushion curves, some conclusions may be made as follows.

(1) The shapes of dynamic cushion curves of honeycomb paperboards with different thickness are similar to that of plastic foams and corrugated paperboard [7,9]. Each piece of the curves shown in Figs 5 to 11 is always concave and upward and has only one minimum value point. 


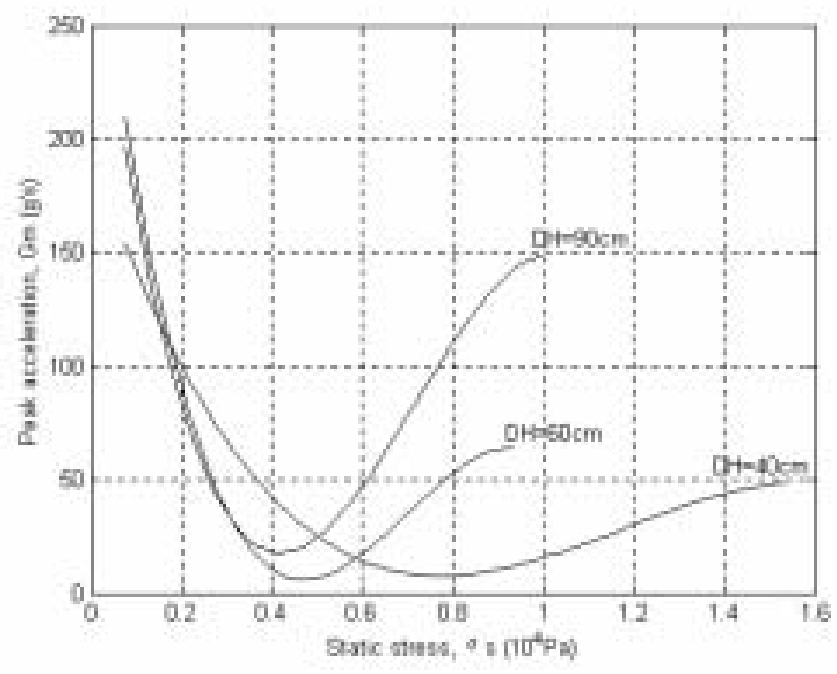

Fig. 8. Dynamic cushion curves of honeycomb paperboard $(T=50 \mathrm{~mm})$.

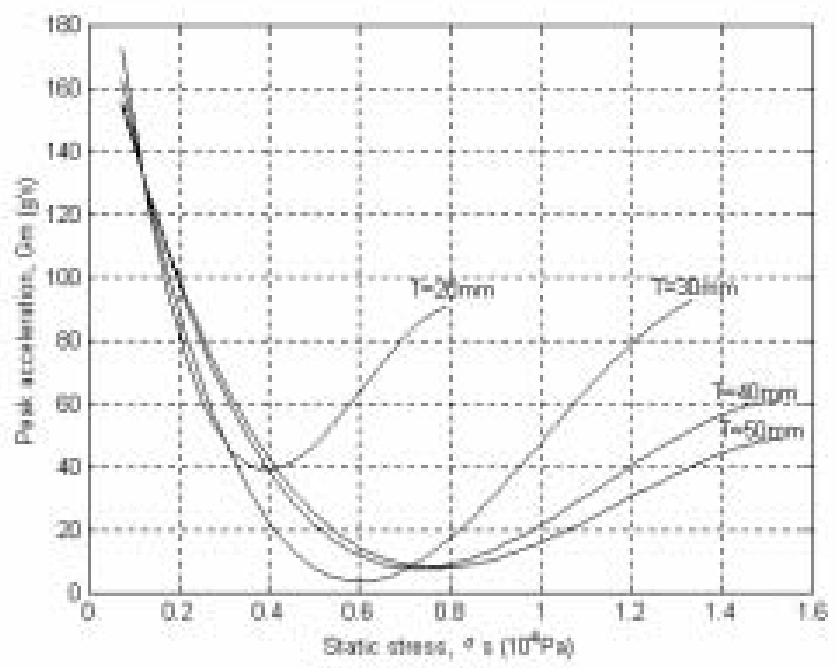

Fig. 9. Dynamic cushion curves of the honeycomb paperboards $(D H=40 \mathrm{~cm})$.

(2) In Figs 5 to 8, for the same thickness of honeycomb paperboard, with the increasing of the drop height, the minimum peak acceleration has a trend to rise, and average peak acceleration increases at the same static stress.

(3) In Figs 9 to 11, for the same drop height, with the increasing of thickness of honeycomb paperboard, the minimum peak acceleration declines, and minimum value point has an excursion along rightward and downward direction.

\section{Vibration transmissibility of honeycomb paperboard}

In this part, the vibration transmissibility of honeycomb paperboards with different thickness is investigated by making vibration tests with slow sine sweep, and the peak frequencies, vibration transmissibility and damping ratios 


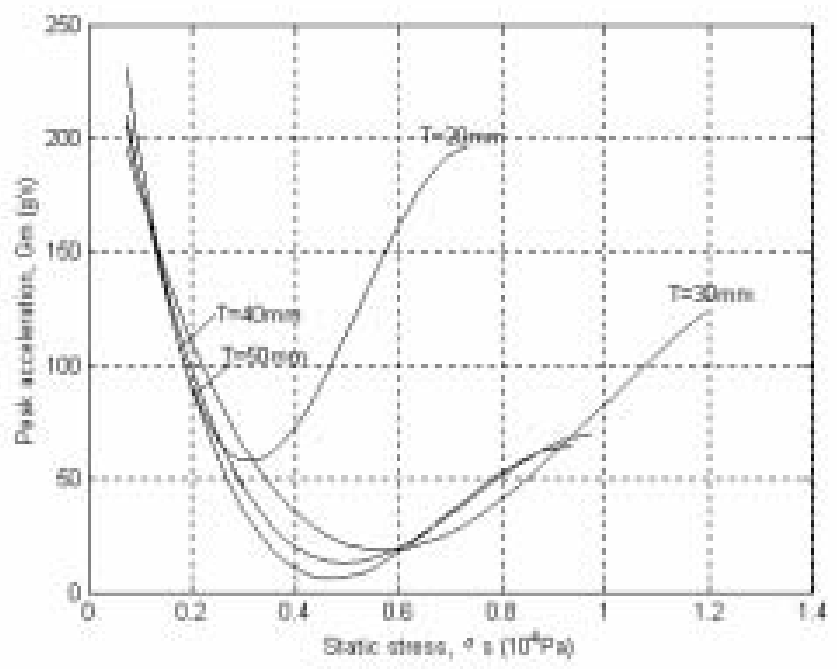

Fig. 10. Dynamic cushion curves of the honeycomb paperboards $(D H=60 \mathrm{~cm})$.

are given.

\subsection{Test results and analysis}

According to the test method described in the ASTM D 4168 standard [2], the vibration tests with slow sine sweep for honeycomb paperboards with different thickness are made, and the peak frequencies, vibration transmissibility and the damping ratios are obtained (see to Tables 2 to 5). It is required to be emphasized that vibration transmissibility $T_{r}$ and peak frequency $f_{r}$ are measured from the vibration tests, yet the damping ratio $\xi$ is approximately estimated by using linear vibration theory. The estimation of damping ratios of honeycomb paperboards with different thickness is discussed in the following Section 4.2.

By comparing the results shown in Tables 2 to 5 , some conclusions of vibration transmissibility of honeycomb paperboard may be reached as follows.

(1) The package cushioning system with honeycomb paperboard has different peak frequencies, yet only one or two of them are primary and other peak frequencies have a little effect on package articles during distribution. For instance, in Table 2, when static stress $3.615 \mathrm{kPa}$ is exerted on honeycomb paperboard with thickness $20 \mathrm{~mm}$, the vibration transmissibility at $200 \mathrm{~Hz}$ is about ten times as much as that of $360 \mathrm{~Hz}$, so peak frequency $200 \mathrm{~Hz}$ should be taken as the first principle mode of vibration, peak frequency $360 \mathrm{~Hz}$ as the second mode, and so on.

(2) In a general case, when the peak frequency shown in Table 2 to Table 5 is more than $350 \mathrm{~Hz}$, the vibration transmissibility is very low, and the honeycomb paperboards may efficiently attenuate vibration with higher frequency during distribution. However, when the peak frequency is less than $350 \mathrm{~Hz}$, the vibration transmissibility is high and the damping ratio is very low.

(3) The static stress exerted on the honeycomb paperboard has influence on vibration transmissibility, which relates to the mechanical behavior of honeycomb paperboard, especially viscoelasticity [4].

Summarizing the above-mentioned conclusion, there are two pieces of regulation that should been taken carefully into account for applying honeycomb paperboard in transport packaging, on one hand, vibration transmissibility must be selected according to the static stress exerted on honeycomb paperboard. On the other hand, the primary peak frequencies must be avoided in package cushioning design of honeycomb paperboard. 
Table 2

Vibration transmissibility of honeycomb paperboard $(T=20 \mathrm{~mm})$

\begin{tabular}{cccc}
\hline$\sigma_{s} / \mathrm{kPa}$ & $f_{r} / \mathrm{Hz}$ & $T_{r}$ & $\xi$ \\
\hline \multirow{3}{*}{1.873} & 160 & 5.604 & 0.091 \\
& 350 & 0.937 & \\
\multirow{3}{*}{4.615} & 200 & 9.429 & 0.053 \\
& 360 & 0.932 & \\
& 230 & 5.966 & 0.085 \\
& 297 & 7.437 & 0.067 \\
& 360 & 4.081 & 0.126 \\
\hline
\end{tabular}

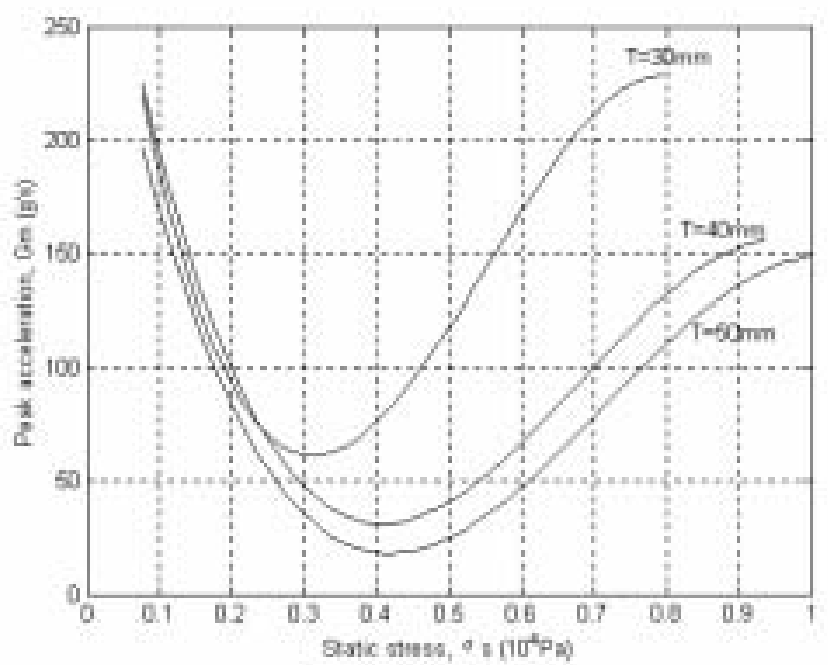

Fig. 11. Dynamic cushion curves of the honeycomb paperboards $(D H=90 \mathrm{~cm})$.

\subsection{Estimation of damping ratio}

Although most package cushioning materials exhibit non-linear characteristics, a brief discussion of linear system will aid in understanding some of the fundamental aspects of vibration as related to packaging considerations [11]. In this section, the linear vibration theory with single degree of freedom system is used to estimate approximately the damping ratios of honeycomb paperboards with different thickness.

For the vibration test system of honeycomb paperboard shown in Fig. 2 (b), the mass block (to represent a packaged item) cushioned on the test specimen can be idealized as the linear single degree of freedom system with viscous damping [11]. The dynamic equation is written as [11]

$$
m \ddot{x}(t)+c \dot{x}(t)+k x(t)=c \dot{y}(t)+k y(t)
$$

where $m$ is the mass of mass block shown in Fig. 2 (b), $c$ and $k$ are the viscous damping coefficient and stiffness coefficient, $x(t)$ is response displacement, and $y(t)$ is excitation displacement.

The vibration transmissibility $T_{r}$ is usually described as a non-dimension ratio of the response acceleration amplitude of the package system in steady-state forced vibration to the excitation acceleration amplitude. Using Fourier transform for the left and right hand sides of Eq. (8), then the vibration transmissibility $T_{r}$ is derived as

$$
T_{r}=\left|\frac{F[\ddot{x}(t)]}{F[\ddot{y}(t)]}\right|=\left|\frac{F[x(t)]}{F[y(t)]}\right|=\sqrt{\frac{1+(2 \xi \lambda)^{2}}{\left(1-\lambda^{2}\right)^{2}+(2 \xi \lambda)^{2}}}
$$

where the symbol 'F[]' represents Fourier transform, $\lambda$ is the frequency ratio

$$
\lambda=\frac{f}{f_{r}}
$$


Table 3

Vibration transmissibility of honeycomb paperboard $(T=30 \mathrm{~mm})$

\begin{tabular}{cccc}
\hline$\sigma_{s} / \mathrm{kPa}$ & $f_{r} / \mathrm{Hz}$ & $T_{r}$ & $\xi$ \\
\hline 2.744 & 202 & 5.966 & 0.093 \\
& 370 & 1.451 & \\
3.615 & 500 & 1.188 & \\
& 146 & 3.342 & 0.157 \\
4.051 & 250 & 0.574 & \\
& 251 & 5.682 & 0.089 \\
& 360 & 3.102 & 0.170 \\
6.228 & 510 & 0.619 & \\
& 150 & 5.454 & 0.092 \\
& 390 & 0.463 & \\
8.406 & 500 & 0.526 & \\
& 197 & 10.120 & 0.049 \\
& 360 & 1.167 & \\
10.584 & 520 & 2.457 & 0.223 \\
& 240 & 9.876 & 0.051 \\
& 360 & 1.948 & 0.299 \\
& 510 & 2.636 & 0.205 \\
\hline
\end{tabular}

Table 4

Vibration transmissibility of honeycomb paperboard $(T=40 \mathrm{~mm})$

\begin{tabular}{ccrc}
\hline$\sigma_{s} / \mathrm{kPa}$ & $f_{r} / \mathrm{Hz}$ & $T_{r}$ & $\xi$ \\
\hline \multirow{2}{*}{3.615} & 150 & 3.984 & 0.130 \\
& 250 & 11.110 & 0.045 \\
4.051 & 180 & 5.721 & 0.088 \\
& 440 & 0.770 & \\
\multirow{2}{*}{6.228} & 200 & 2.670 & 0.202 \\
& 250 & 4.377 & 0.117 \\
& 360 & 2.424 & 0.226 \\
10.584 & 200 & 9.395 & 0.054 \\
& 350 & 1.080 & \\
\hline
\end{tabular}

where $f_{r}$ is the peak frequency, and $\xi$ is the damping ratio

$$
\xi=\frac{c}{4 \pi m f_{r}}
$$

It is clear that vibration transmissibility $T_{r}$ has close relationship with frequency ratio $\lambda$ and damping ratio $\xi$. When resonance takes place, $\lambda \approx 1$. From Eq. (9), damping ratio $\xi$ may be estimated as

$$
\xi=\frac{1}{2} \sqrt{\frac{1}{T_{r}^{2}-1}}
$$

If $T_{r} \gg 1$, damping ratio $\xi$ may be approximately calculated by the following expression

$$
\xi \approx \frac{1}{2 T_{r}}
$$

By using Eqs (12) and (13), and the vibration transmissibility $T_{r}$ shown in Tables 2 to 5, the damping ratios $\xi$ are estimated and given in the last column of Tables 2 to 5 .

\section{Conclusion}

Honeycomb paperboard belongs to a kind of environmental-friendly package cushioning material with honeycomb sandwich structure, possessing an attractive prospective in transport packaging. This paper deals with the characterization of properties of honeycomb paperboard relevant to its application for protective packaging in transportation, such as dynamic cushion curves and vibration transmissibility. The waves of shock acceleration are similar 
Table 5

\begin{tabular}{cccc}
\multicolumn{4}{c}{ Vibration transmissibility of honeycomb paperboard $(T=50 \mathrm{~mm})$} \\
\hline$\sigma_{s} / \mathrm{kPa}$ & $f_{r} / \mathrm{Hz}$ & $T_{r}$ & $\xi$ \\
\hline 4.051 & 200 & 5.231 & 0.097 \\
& 310 & 1.473 & \\
6.228 & 170 & 4.842 & 0.114 \\
& 250 & 9.775 & 0.051 \\
8.406 & 360 & 1.544 & \\
& 200 & 5.784 & 0.088 \\
& 420 & 1.047 & \\
10.584 & 490 & 1.448 & \\
& 150 & 1.697 & 0.365 \\
& 210 & 6.044 & 0.084 \\
& 360 & 1.296 & \\
\hline
\end{tabular}

to half-sine pulses, and the shapes of dynamic cushion curves are always concave and upward, and each piece of dynamic cushion curves has only one minimum value point. From the vibration tests with slow sine sweep, the peak frequencies and vibration transmissibility are measured and used to estimate damping ratios. When peak frequency is more than $350 \mathrm{~Hz}$, vibration transmissibility is very low. The results show that honeycomb paperboard posses shock absorbing characteristics for a single impact (drop), but it couldn't protect products from consecutive impacts and it may efficiently attenuate vibration with higher excitation frequency during distribution. All the works provide basic data and curves relevant to its application for protective packaging in transportation.

\section{Acknowledgements}

This work is supported by the Education Department of Shaanxi Province Scientific Research Foundation under the Grant 00JK221 and the National Natural Science Foundation of China under the Grant 10272087, and the test specimens are supplied by Xi'an Yongcheng Honeycomb Paperboard Limited Company.

\section{References}

[1] ASTM D 1596 Standard Test Method for Shock Absorbing Characteristics of Package Cushioning Materials.

[2] ASTM D 4168 Standard Test Method for Vibration Transmissibility of Package Cushioning Materials.

[3] G. Burgess, Generation of Cushion Curves from One Shock Pulse, International Journal of Packaging Technology and Science 7(2) (1994), 169-173.

[4] Guo, Yanfeng, Xu, Wencai and Pan, Songnian, Applied study on honeycomb paperboard and cushion, 13th IAPRI World Conference Proceedings on Packaging, East Lansing, 2002, pp. 782-787.

[5] J. Kirkpatrick and M. Sek, Replacement of Polymeric Cushioning with Corrugated Fiberboard-case Study, 10th IAPRI World Conference on Packaging, Melbourne, 1997, pp. 267-276.

[6] K.M. Liew and C.S. Lin, Analysis of Dynamic Response of Delaminated Honeycomb Panels, International Journal of Composite Structure 39(1-2) (1997), 111-121.

[7] J. Miltz, O. Ramon and S. Mizrahi, Mechanical Behavior of Closed Cell Plastic Foams Used as Cushioning Materials, International Journal of Applied Polymer Science 38(2) (1989), 281-290.

[8] R.D. Mindlin, Dynamics of Package Cushioning, Bell System Technical Journal 24(7-10) (1945), 352-461.

[9] M. Sek and J. Kirkpatrick, Characteristics of Corrugated Fiberboard as a Cushioning Material in Protective Packaging, 10th IAPRI World Conference on Packaging, Melbourne, 1997, 257-266.

[10] M. Sek and J. Kirkpatrick, Prediction of Cushioning Properties of Corrugated Fiberboard from Static and Quasi-dynamic Compression Data, International Journal of Packaging Technology and Science 10(2) (1997), 87-94.

[11] U.S. Department of Defense, Military Standardization Handbook, Package Cushioning Design, MIL-HDBK-304B, 1978.

[12] H.X. Zhu and N.J. Mills, The In-plane Non-linear Compression of Regular Honeycomb, International Journal of Solids and Structures 37(13) (2000), 1931-1949. 

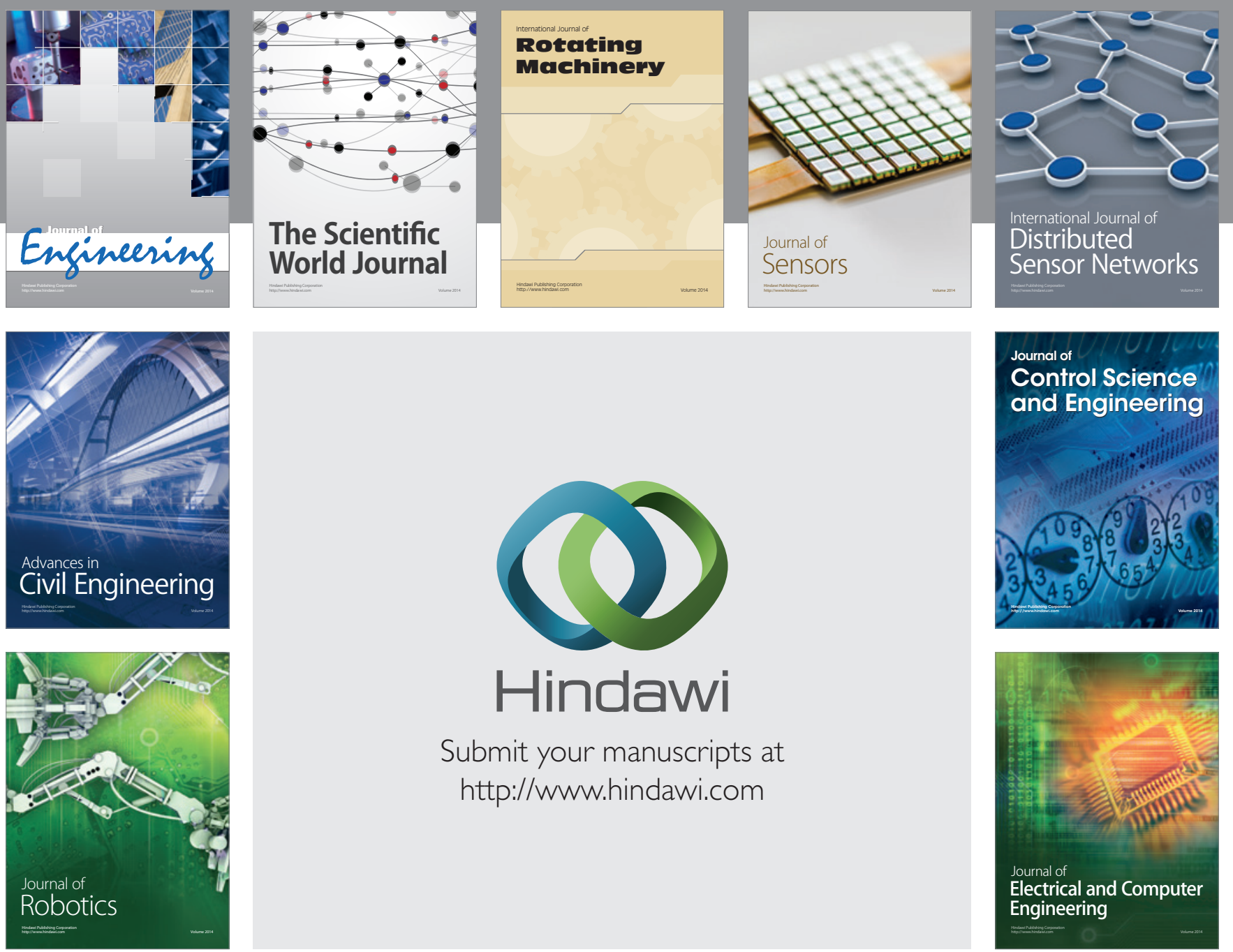

Submit your manuscripts at

http://www.hindawi.com
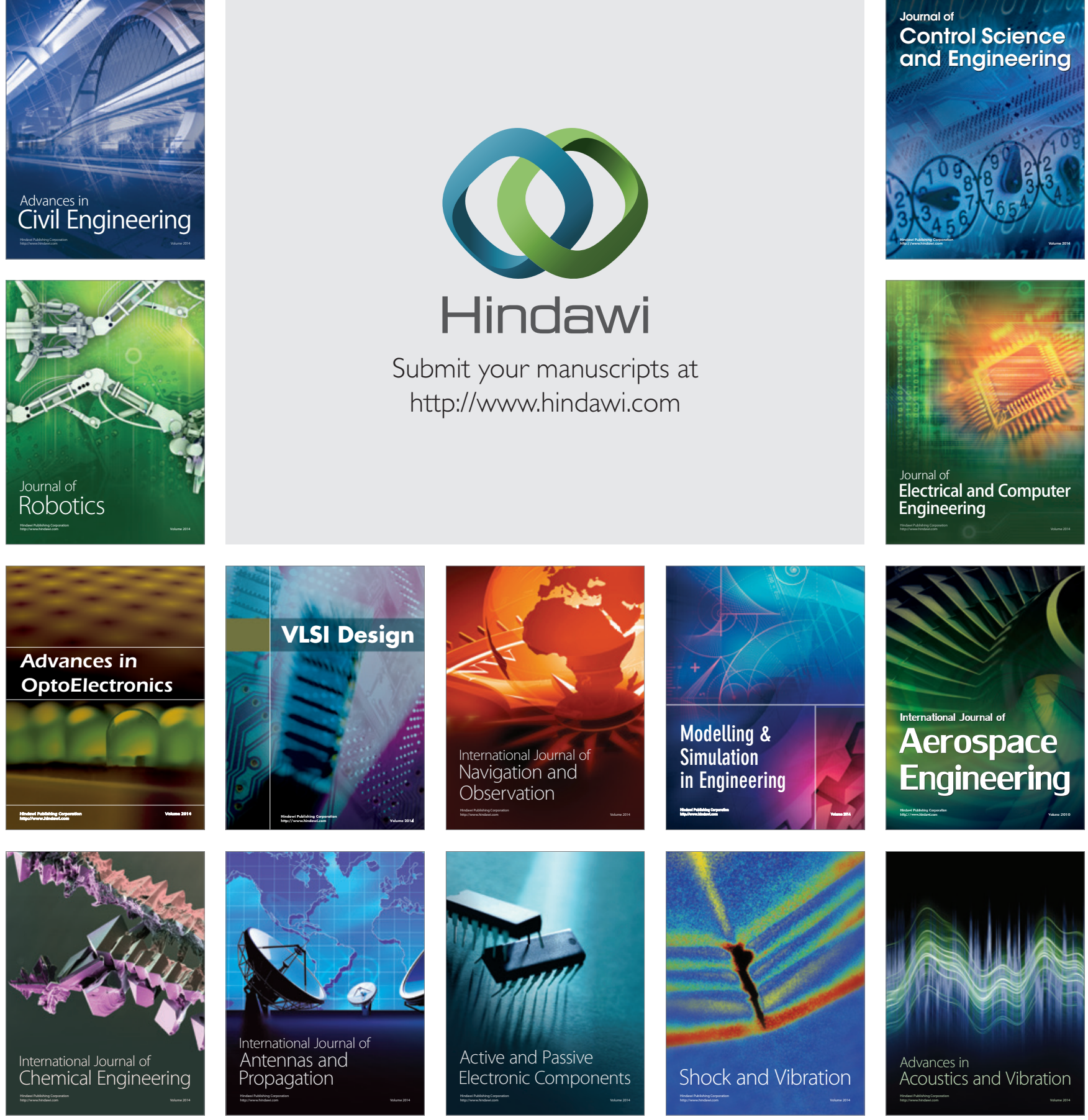